\title{
Smart Cities and Cultural Heritage - A Review of Developments and Future Opportunities
}

\author{
Ann Borda \\ Health and Biomedical Informatics Centre \\ The University of Melbourne \\ Melbourne, Australia \\ aborda@unimelb.edu.au
}

\author{
Jonathan $\mathrm{P}$. Bowen \\ London South Bank University \\ \& Museophile Limited, UK \\ www.jpbowen.com \\ jonathan.bowen@/sbu.ac.uk
}

\begin{abstract}
This paper reviews a cross-section of international developments in smart cities and their implications for the cultural heritage sector. A main focus of the paper is an assessment of selected case studies in the cultural heritage sector exploring the use of smart platforms and visualisation technologies. The results highlight a particular set of current challenges, as well as providing scope for identifying future opportunities in developing smart cultural heritage services.
\end{abstract}

Smart cities. Smart heritage. Smart culture. Smart museums. Visualisation. Internet of Things. Augmented reality.

\section{INTRODUCTION}

Soja \& Kanai (2006) use the terms "global city region" to refer to "a new metropolitan form characterised by sprawling polycentric networks of urban centres ..." Such networks are becoming identified with both the potential and the reality of 'smart' city infrastructures of connected transportation, financial, energy, health, information and cultural systems.

There are numerous definitions of a "smart city" across the literature with little consensus (Angelidou 2015) and there are many technical issues involved (Palomar et al. 2016). For the purposes of this paper, the definition provided by the (ISO/IEC 2014) is considered an appropriately inclusive one, that is:

\footnotetext{
The "smartness" of a city describes its ability to bring together all its resources, to effectively and seamlessly achieve the goals and fulfil the purposes it has set itself...[It] enables the integration and interoperability of city systems in order to provide value, both to the city as a whole, and to the individual citizen. This integration further enables potential synergies to be exploited and the city to function holistically, and to facilitate innovation and growth.
}

In the context of such values and goals, there is a global movement in the implementation of smart cities which was catalysed in the Global Forum World Foundation for Smart Communities in 1997. In particular coordinated strategies and standards for smart city implementation are increasingly pervasive and being adopted at national and industry levels. For example, the UK Department of Business, Innovation and Skills commissioned BSI in 2012 to develop a standards strategy for smart cities in order to accelerate and minimise risks in the implementation of smart cities in the UK. In 2011, the European Commission initiated the European Innovation Partnership on Smart Cities and Communities (EC 2015). In China, comparable initiatives have been established, such as the China Strategic Alliance of Smart City Industrial Technology Innovation. In the United States, the Federal Smart Cities and Communities Task Force is seeking to embed new digital technologies into city and community infrastructures and services. The Australian government has similarly launched a national Smart Cities Plan in 2016 aimed at positioning Australian cities to succeed in the digital economy (Australian Government 2017).

Among individual cities themselves, there are examples of smart city plans that are being developed at local and municipal government level. One example is the GrowSmarter (2015) initiative, a collaborative EU funded smart city project, focusing on sustainable solutions to economic, social and environmental issues. The project involves what are termed "Lighthouse Cities" of Stockholm, Cologne and Barcelona. It aims to integrate and demonstrate twelve smart solutions to energy, mobility and infrastructure in collaboration with twenty industrial partners, and importantly the project is intended to create a 
platform for sharing knowledge and experience. Industry involvement in smart city developments is especially key to such partnerships, and in supporting the technological enablers and connected platforms that underpin smart city infrastructures. Multinational communications and IT companies, Cisco and Nokia are among the industry players who are developing strategic White Papers about the platform components of a successful smart city, and partnering with cities on pilot implementations (Cisco 2014, Nokia 2017).

Across these standards and strategies is a shared vision to position communities at all scales to have equitable access to connected smart services that can enhance the sustainability and quality of life, improved health and safety, and economic prosperity. Smart cities can help enable virtual collaboration of communities (Borda \& Bowen 2011).

In the context of this proposed paper, the citizens of a smart city are potential participants in its governance and in the evolving development of smarter services, including those related to accessing and preserving cultural heritage and the arts. Now, however, there are few visible examples of smart cultural initiatives integrated with smart city developments at a pilot or a conceptual level. There is consequently a need to understand how populations can be supported by local capacities and smarter cultural cities and regions, using advanced information systems, visualisation, and applications.

\section{SMART CULTURAL HERITAGE}

The concept of "smart cultural heritage," according to researchers of the EU funded DATABENC (Distretto ad Alta Tecnologia per i Beni Culturali) initiative, is about digitally connecting institutions, visitors, and objects in dialogue. Smart heritage focuses on adopting more participatory and collaborative approaches, making cultural data freely available (open), and consequently increasing the opportunities for interpretation, digital curation, and innovation. This offers potential and unprecedented access to cultural artefacts and experiences across distances, in which cultural consumers are no longer passive recipients (Amato et al. 2013, Angelaccio et al. 2012, Chianese et al. 2015, Roffia et al. 2005, Garcia-Crespo 2016, Yovcheva 2014).

As described in the Europeana White Paper on smart cities, "cultural heritage defines our identity and our communities. Sharing our past in smart city initiatives has the potential to promote social cohesion and increase innovation and tourism (Europeana 2015).
In this way, smart cultural heritage is strongly associated with the identity of place and communities through smart technologies, knowledge and participation. It is not surprising that the cultural heritage sector has been working within smart requirements for many years due to the inseparable association with location and identity (Chianese et al. 2013). Projects such as the prototype multimedia guide developed for the archaeological site of ancient Olympia in Greece, ARCHEOGUIDE (Augmented Reality-based Cultural Heritage On-site Guide) provided augmented reconstructions of the ancient ruins and audio information (Vlahakis et al. 2001). ARCHEOGUIDE supported a context awareness visitor application, i.e., a location-based application, in which a user's location is identified through a sensing device, and provides the user with information bound to that specific location and the physical objects in the surroundings. The development of context-aware services has been pervasive in demonstrator applications in the cultural heritage areas, not least focused on forms of digital data and user defined interactions (Chianese et al. 2015, Roffia et al. 2005).

With the socio-technical rise of the mobile phone, museums and galleries worldwide developed mobile apps that visitors could download onto their own device and create self-guided tours. The National Gallery in London was one of the first museums to develop LoveArt - an iPhone app launched in 2009 (Lagoudi \& Sexton 2010).

\subsection{Enabling Technologies}

In the context of both smart cities and smart cultural heritage, the smartness requirements of each are equally aligned to new intelligent and contextualised services. Across the literature, these services are generally made possible by a common set of key technologies that are becoming ubiquitous and inseparably identified with the realization of smart developments.

\subsubsection{Internet of Things (IoT)}

loT is a vast network of devices connected to the Internet, including smart phones and tablets and any device and/or object with embedded sensors that are capable of transmitting data across networks. These "things" collect and exchange data across networks, often in real time. The emergence of loT as a global infrastructure of the digital information society is rapidly becoming identified as the enabler of smarter cities. Gartner has predicted that by 2020 , over 20 billion connected things will be in use (Gartner 2017).

\subsubsection{Cloud computing}

Cloud computing is the delivery of on-demand computing resources, ranging from applications to 
data centres, over the Internet. It offers an agile and flexible framework to connect sites, people and applications securely and to enable the sharing of information and resources with economies of scale. Cloud computing and the loT both serve to increase efficiency in everyday tasks and have a complementary relationship. The loT, by its nature, will generate large amounts of data, and cloud computing provides a way of efficiently allocating resources in different areas, making it more feasible to access large quantities of loT data, for instance, via cloud services.

\subsubsection{Wireless Sensor Network (WSN)}

WSN is a wireless network consisting of spatially distributed autonomous devices using sensors, often to monitor surrounding context, physical or environmental conditions. The innovation of WSN is that it acts as a virtual layer where data about the physical world can be accessed by any computing system.

\subsubsection{Mobile broadband}

Loosely defined as the wireless Internet accessible through a portable modem, mobile phone, USB wireless modem, tablet or other mobile devices, including those using wi-fi protocols. Recently in 2016, StatCounter reported that $51.3 \%$ of web browsing worldwide took place via mobiles and tablets, overtaking desktop browsing for the first time. Google has also adopted mobile friendly algorithms with the recent inclusion of a mobile usability testing tool as part of its suite of tools.

\subsubsection{Short range wireless}

The characteristics of short-range wireless technologies make these preferred technology platforms for the development of smart and location-aware environments. Two emerging technologies are:

(i) Bluetooth Low Energy (BLE) is a subset of Bluetooth wireless technology designed for power efficient applications. It allows physical objects to exchange data wirelessly and is being applied in security, home entertainment, and beacon technologies, for example. Most mobile operating systems natively support BLE.

(ii) NearField Communication (NFC) enables short range wireless communication between devices or objects, e.g., a portable device, such as a mobile, iPad or smartphone, when they are touched together, or brought within a few centimetres of the other. NFC technology is identified with contactless Radio Frequency IDentification (RFID) technology.

\subsection{Visualisation Technologies}

Visualisation is an important component in shaping smart cultural heritage developments and in providing a tangible interface between the user and the enabling and often invisible technologies lying beneath (Li et al. 2016). Importantly, visualisation is a proven method of increasing the effectiveness of information and knowledge access and representation in cultural heritage interpretation and user interaction. In an increasingly contextaware environment, visualization importantly provides an opportunity to represent different forms of digital data regardless of source, and equally provides users the means to explore a large set of data points simultaneously. A growing list of devices built with natural user interfaces (NUIs) that accept input in the form of taps, swipes, and touch, such as tablets and smartphones, are among the devices that are further reliant on adaptive visualization to simplify the user experience.

Visualisation technologies themselves are varied, from simple to advanced, such as simple infographics to $3 \mathrm{D}$ modeling. Data visualisation is an underpinning process across these, to communicate underlying data or information by encoding it as visual objects. An infographic (information graphic), for example, is a type of data visualisation.

The process is intended to make complex data more accessible, understandable, and usable. In smart environments with potentially massive amounts of IoT generated data, data visualisation will be critical to sense-making of the data through data analysis and its application to smart services.

In a cross-section of smart cultural heritage projects (see: section 3. Characterising Smart Cultural Heritage), the visualisation technologies that are presently identified in this study with smart cultural developments, include:

\subsection{1 $3 \mathrm{D}$ visualization}

The three-dimensional (3D) visualization of data as represented in multi-dimensional planes, usually as a 3D model frame or object. 3D visualization is an interchangeable term with 3D graphics, 3D rendering, and/or computer-generated imagery (CGI), as well as the 3D software generated files used for 3D printing.

\subsubsection{Geovisualisation}

Geo(spatial) visualisation refers to a set of tools and techniques supporting the analysis of geospatial data using interactive visualisation, for example, in which maps can be overlaid with additional layers of information based on cartographic coordinates and/or Geographic information systems (GIS) data. In this way, geovisualisation allows for the ability to explore 
different layers of a map at various scales and across thematic layers, and to change the visual appearance of the map, e.g., Google Earth applications.

\subsubsection{Augmented Reality (AR)}

$A R$ is the interaction of superimposed data, graphics, audio and other sensory enhancements over a real-world environment that is displayed in real time. AR browsers deliver geospatial and attribute information about physical objects through spatially linked virtual annotations, and this characteristic has brought AR into the domains of travel and cultural tourism, particularly in supplementing tour maps, guidebooks, or simple list-based mobile applications. The recent launch of Pokémon Go, a game mainly designed around maps and real world locations, entices players to find and catch characters from Nintendo's Pokémon game, has heightened public awareness of the potential of AR (Bego 2016)

\section{CHARACTERISING SMART CULTURAL HERITAGE}

For the purposes of this study a cross-selection of case studies were brought together to examine more closely shared and different characteristics that might be identified with smart cultural heritage developments, as well as gaps and opportunities. Case studies were selected, for example, based on available information in English, evidence of embedded use within an organisational context, location-based application, integration of types of enabling technologies and visualisation (as outlined in sections 2.1 and 2.2), as well as examples from indoor and outdoor settings. Where possible, examples were provided from museum, gallery, and archaeological sites.

Exclusion criteria included non-English language examples (where minimal or no translation exists), research projects, case studies prior to 2012 , audio guides, and list-based mobile or web applications, or mobile apps which are not free to download.

Each case study was grouped by a primary 'function' in one of three areas:

- Preservation and Reconstruction

- Digital Trail

- Exhibition Tour

In this paper, the distinction between a Digital Trail and Exhibition Tour is premised on the general nature of an exhibition as a themed display in a designated or bounded space, and often it is a time-limited or temporary event. A digital trail may allow the visitor to traverse several exhibitions, permanent collections and/or different physical spaces, for example. The three categories are also not exclusive, and examples can inevitably fall into more than one category. Pure Land, for example, arose as a digital reconstruction of a Tang Dynasty Buddhist cave that could be explored through exhibition and thus minimise risk and exposure to visitors to the actual cave.

Another consideration was the function of education. This was seen as a pervasive function across the examples and did not warrant a separate category for this short paper. Finally, suitable examples arising from libraries, as potential key participants in smart cultural heritage, were not located for this brief study. This also warrants a future investigation.

\section{1. Preservation and Reconstruction}

\subsubsection{Pure Land: the Mogao Grottoes at Dunhuang}

City University, Hong Kong, China http://alive.scm.cityu.edu.hk/projects/alive/pureland-ii-2012/

Pure Land immerses visitors in a $360^{\circ}$ immersive and Augmented Reality (AR) designed interactive space to explore the heritage of Tang Dynasty era Buddhist cave temples hewn in rock, and housing painted murals, statues and architectural monuments. This UNESCO World Heritage site, also known as the Caves of the Thousand Buddhas, is located at Dunhuang, a small town in northwestern China. The datasets of Cave 220, with its richly narrative murals, are the subject for Pure Land.

$A R$ is among the technologies that enhance the cave's architectural and photographic representations with animation, 3-D modelling, pictorial re-colouring, digital enlargement and sound design.

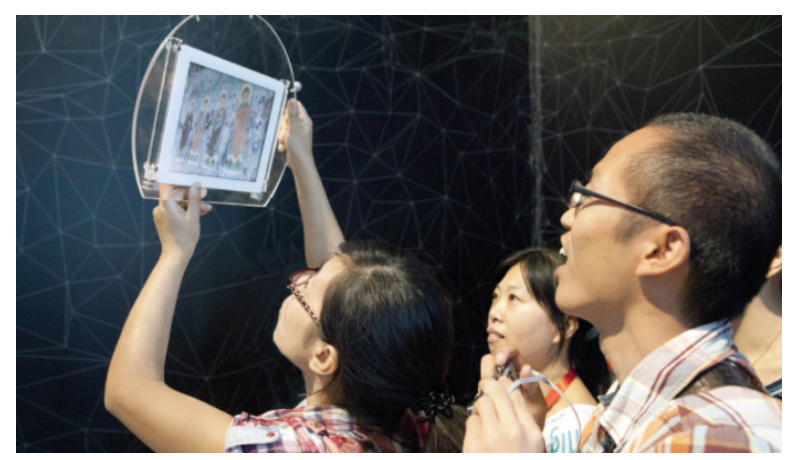

Figure 1: Pure Land AR [2012], Shanghai Biennale, September 2012 to March 2013. Image (C) Applied Laboratory for Interactive Visualization and Embodiment, City University Hong Kong.

\subsubsection{Rekrei}

Rekrei.org (https://rekrei.org/) 
The destruction of the Mosul Museum in Iraq led to the formation of Project Mosul, later renamed as Rekrei. Rekrei is a crowdsourced project to collect photographs of monuments, museums, and artefacts damaged by natural disasters or human intervention, and to use this data to create $3 \mathrm{D}$ representations to help preserve a global, shared, human heritage. Thousands of photographs uploaded to Project Mosul by local residents, tourists, and American soldiers deployed in the region during the Iraq war resulted in a collective reconstruction supported by the Economist, entitled RecoVR Mosul.

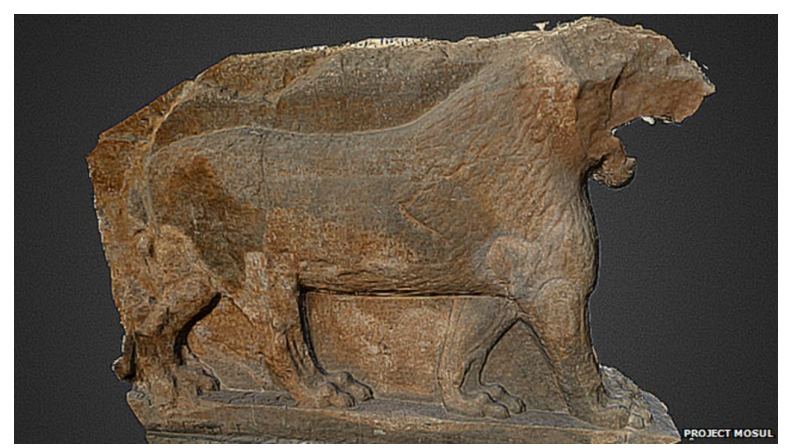

Figure 2: 3D rendering of the Lion of Mosul. Project Mosul, 2015.

\subsubsection{Zamani Project}

University of Cape Town, South Africa http://www.zamaniproject.org

The University of Cape Town and collaborators have spent over a decade documenting Africa's most important cultural and heritage buildings, sites and landscapes. The project teams have utilised data generated from GIS, 3D computer models and other spatial data. The site acts as a data repository with geovisualisations, model reconstructions, and panoramas.

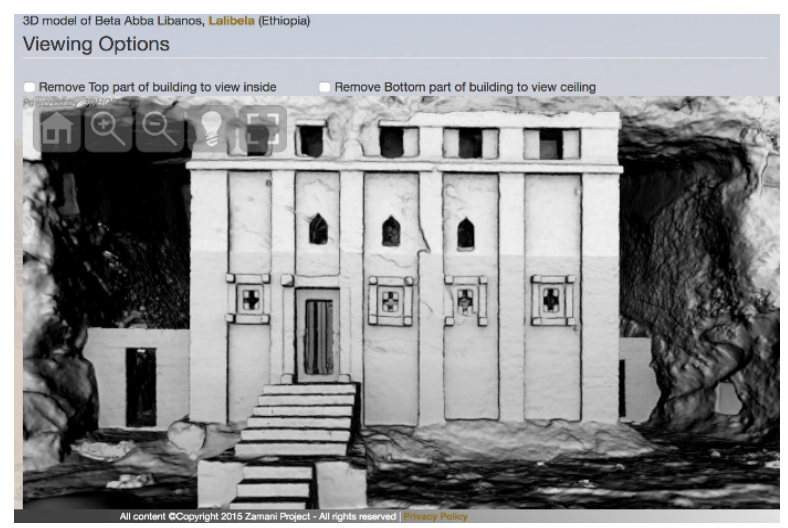

Figure 3: Navigable 3D model of Beta Abba Libanos, Ethiopia. Source: Zimani Project website, (c) 2015.

Since the completion of the project in 2015, the complete geospatial datasets are currently held with Aluka Heritage which is accessible through JSTOR to subscribed institutions.

\subsection{Digital Trail}

\subsubsection{The O-device}

Museum of New and Old Art (MoNA)

Hobart, Tasmania, Australia

https://mona.net.au/museum/the-o

The O-device is a digital trail guide that uses an internal positioning system to locate the visitor in each of the exhibition spaces of MoNA. The $O$ device presents different curatorial texts, video and audio about each of the artworks in the vicinity of the visitor. Content can be personalised and saved by the visitor for access later through a web browser. The visitor can directly download the $\mathrm{O}$ app to an iPhone, e.g., through wi-fi within MoNA, or use a MoNA O device. The $O$ app can also be used off site.

\subsubsection{StreetMuseum Mobile App}

Museum of London, London, UK http://www.museumoflondon.org.uk/Resources/app lyou-are-here-app/home.html

Streetmuseum is an AR enabled App for smartphones for exploring a variety of historic landmarks and unique sights of London. The GPS in a user's mobile device identifies the user's location in the city and then overlays a historic image onto the camera view, such as Blackfriars station in 1930, Victoria Station in 1950, and London's skyline seen from Tower Bridge. The App also allows users to select a destination from a London map. The user can access and download images, photos and paintings of the location from the Museum of London collections. The App can be downloaded to an iOS or an Android device and used from home and as a post visit experience.

\subsubsection{Paisatge: Barcelona Landscape Webapp}

Barcelona City Council (Ajuntament de Barcelona) http://paisatge.bcn.cat/en/

Meaning 'landscape' in Catalan, Paisatge is an interactive Webapp that provides a digital trail around the City of Barcelona to discover the city's architectural and landscape features. The App allows for real-time discovery of landmarks in the proximity of the visitor and sends alerts of events in the vicinity.

The Webapp is designed for mobile devices and supports functions, such as geolocation and touch browsing. Visitors can share, recommend, and save information ("bookmarking") for a post-visit experience (Filippini-Fantoni \& Bowen 2007). The App and content can be accessed from any mobile device with an Internet connection. 


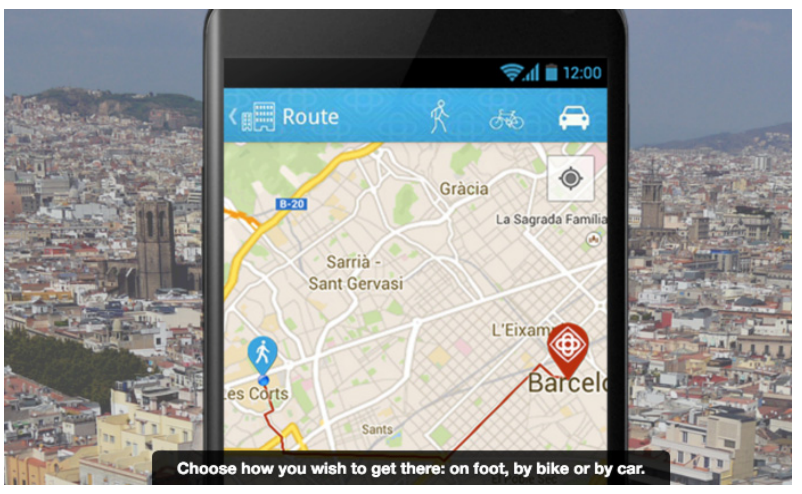

Figure 4: Image of Mobile device showing BCN Landscape app in use. ${ }^{\odot}$ BCN website, 2017.

\subsubsection{Journey of Inspiration Mobile App}

Canadian Museum for Human Rights Winnipeg, Manitoba, Canada https://humanrights.ca/visit/tours/mobile-app

The Canadian Museum for Human Rights Mobile App Journey of Inspiration is a fully accessible selfguided tour using audio, images, text and video, interactive map, online ticketing, and information to help plan the visit. The device works through lowfrequency "iBeacons" to over 120 Universal Access Points (UAPs) located throughout the Museum. Included is an interactive panorama (AR) feature and an interactive "mood meter" feature allowing the visitor to share how they feel and see the results of others as they move through the Museum. Users can download the App prior to the visit, use the Museum's Wi-Fi to download the app to iOS/Android device, or borrow a Museum preloaded device.

\subsubsection{The Pen}

Cooper Hewitt, Smithsonian Design Museum New York, USA https://labs.cooperhewitt.org/tag/the-pen/

The Pen is a high-tech device that enables visitors to collect "objects" from around the galleries and create their own designs on interactive tables. The Pen uses NFC-reading technology. At the end of a visit, objects collected or designed by the visitor using the Pen are accessible online through a unique web address printed on the visitor's ticket. These can be shared online and stored for later use in subsequent visits.

\subsubsection{The Collection Wall}

Cleveland Museum of Art (CMA), Ohio, US http://www.clevelandart.org/gallery-one/collectionwall

The Collection Wall, standing 5 feet by 40 feet, is a multi-touch MultiTile system that allows multiple users to explore the digital collections of CMA in real time and thematically. The Wall is updated every few minutes with high-resolution images, new exhibition data, and the frequency with which each artwork has been "favourited" on the Wall and/or from the ArtLens App (the CMA collections mobile application). Visitors can save favourites to an iOS/Android device on the ArtLens App directly from the Wall using a docking station (Bluetoothenabled). ArtLens can be used on-site or off-site.

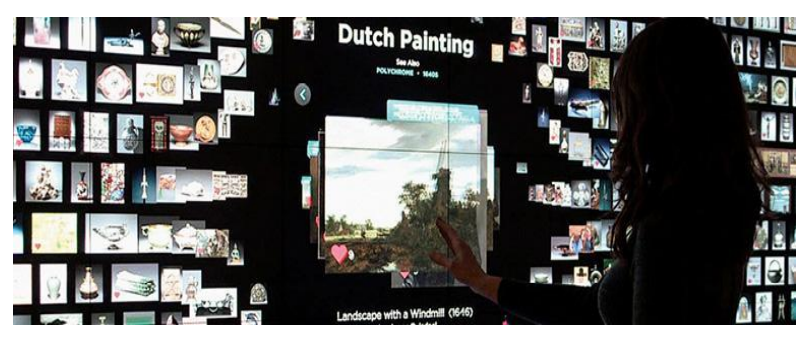

Figure 5: The Collection Wall.

( ) Cleveland Museum of Art, 2017.

\subsection{Exhibition Tour}

\subsubsection{Skin and Bones}

National Museum of Natural History, Smithsonian Institution, Washington, D.C., USA

http://naturalhistory.si.edu/exhibits/bone-hall/

The Bone Hall is a comparative anatomy exhibit that opened the Smithsonian's first museum in 1881. Skin \& Bones is an AR application that superimposes a virtual world onto physical objects held in the gallery, by directing the camera of a device towards the object. With the App, visitors also test natural history skills playing games, such as identifying a bat by its distinctive call. Skin \& Bones is compatible with iPhone, iPad, and iPod touch.

\subsubsection{Ai WeiWei 360}

Royal Academy, London, UK https://www.royalacademy.org.uk/exhibition/aiweiwei-360

The Ai WeiWei application provides the user with an immersive tour related to the $A i$ WeiWei exhibition held at the Royal Academy London, 20 January 2016 - 12 January 2017. The exhibition was captured in photorealistic stereoscopic 3D and consists of navigable $360^{\circ}$ imagery, video and audio channels. A user can access the tour from the Royal Academy website, alongside commentary from the exhibition's curators and interviews with the artist. The user can explore the App from a desktop, a tablet or a smartphone device using an Internet browser. It is also available to use on both Samsung Gear VR and Google Cardboard. 


\subsubsection{Ultimate Dinosaurs: Giants from Gondwana}

Royal Ontario Museum, Toronto, Canada https://www.rom.on.ca/en/exhibitionsgalleries/exhibitions/past-exhibitions/ultimatedinos/augmented-reality

The ROM Ultimate Dinosaurs App was designed for the 2012 Exhibition and made available for iPhone, iTouch and iPad to allow users to experience dinosaurs with AR enabled content and functions (e.g., scanning skeletal casts to bring them alive). The App could be used both in the exhibit itself and also outside by interacting with selected ROM advertising points across the Greater Toronto Area, including transit shelters and print advertising.

\section{ANALYSIS OF CHARACTERISTICS}

The case studies were each reviewed and compared in terms of enabling smart technologies and visualisation types (Table 1), and other characteristics which arose in the comparison process. The analysis was based on available published information. Direct experience of the profiled apps and systems was only possible for a handful of the selected studies, but overall this was a minimal contributing factor in reviewing characteristics of "smart" components, rather than the more detailed considerations of user experience.

\begin{tabular}{|c|c|c|c|c|c|c|}
\hline $\begin{array}{l}\text { Preservation \& } \\
\text { Reconstruction }\end{array}$ & WSN & Broadband & $\begin{array}{c}\begin{array}{c}\text { Short range } \\
\text { wireless }\end{array} \\
\end{array}$ & 3D Vis & GeoVis & AR \\
\hline Pure Land 360 & $\checkmark$ & $\checkmark$ & $\checkmark$ & $\checkmark$ & $\checkmark$ & $\checkmark$ \\
\hline Rekrei & & $(\checkmark)$ & & $\checkmark$ & $\checkmark$ & $(\checkmark)$ \\
\hline Zamani Project & & $(\sqrt{ })$ & & $\checkmark$ & $\checkmark$ & \\
\hline Digital Trail & WSN & Broadband & $\begin{array}{c}\text { Short range } \\
\text { wireless }\end{array}$ & 3D Vis & GeoVis & AR \\
\hline O-Device & $\checkmark$ & $\checkmark$ & $\checkmark$ & & $\checkmark$ & \\
\hline StreetMuseum & $\checkmark$ & $\checkmark$ & & $\checkmark$ & $\checkmark$ & $\checkmark$ \\
\hline Paisatge & $\checkmark$ & $\checkmark$ & & & $\checkmark$ & \\
\hline Journey of Inspiration & $\checkmark$ & $\checkmark$ & $\checkmark$ & $\checkmark$ & $\checkmark$ & $\checkmark$ \\
\hline The Pen & $\checkmark$ & $\checkmark$ & $\checkmark$ & $\checkmark$ & $\checkmark$ & \\
\hline The Collection Wall & $\checkmark$ & $\checkmark$ & $\checkmark$ & & $\checkmark$ & \\
\hline Exhibition Tour & WSN & Broadband & $\begin{array}{c}\text { Short range } \\
\text { wireless }\end{array}$ & 3D Vis & GeoVis & AR \\
\hline Skin and Bones & $\checkmark$ & $\checkmark$ & $\checkmark$ & $\checkmark$ & $\checkmark$ & $\checkmark$ \\
\hline Ai WeiWei 360 & & $\checkmark$ & & & $\checkmark$ & $(\checkmark)$ \\
\hline Ultimate Dinosaurs & $\checkmark$ & $\checkmark$ & $\checkmark$ & $\checkmark$ & $\checkmark$ & $\checkmark$ \\
\hline
\end{tabular}

Note: $(\checkmark)$ Accessible through the technology or incorporating features of the technology, but not a specific application for that technology.

Table 1: Summary overview of use of enabling technologies and visualisation types across the selected case studies.

\subsection{Enabling technologies}

At the time of this paper, there are no published standards specific to smart cultural heritage projects as there is available for smart cities, such as those developed by ISO/IEC or the IEEE Smart Cities Initiative (IEEE 2017). However, there are some advances towards developing platforms for smart cultural heritage utilising enabling technologies that underline smart city implementation. Among the enabling technologies, mobile broadband is pervasive across the case study examples in use and/or in access. The cultural heritage sector has been an early adopter of mobile technologies in user engagement and the visitor experience in the development of mobile apps. It is also the most accessible and available of the technologies to the broadest spectrum of users, irrespective of their location (Roffia et al. 2005, Casella \& Coelho 2013, Yovcheva 2012, Yovcheva et al. 2014).

Wireless Sensor Networks (WSN) are another layer of infrastructure that is increasingly common supporting different smart scenarios. Smartphone tours and devices that are context-aware figure in most of the examples, such as the indoor digital trails of the O-Device and Journey of Inspiration, and city trails of Paisatge and StreetMuseum.

The application of NFC technology provides a more fine-grained context-awareness that allows users to receive customised information and a more realistic experience in close proximity, e.g., users can read or listen to comprehensive guides about landmarks they discover, while watching animations or playing games. The Pen at Cooper Hewitt builds on NFC reading technology to enable personalised and individual interaction. The use of BLE enabled beacons across the Canadian Museum for Human Rights, supports a digital trail that is layered with narrative and augmented reality. 120 universal access points also provides improved visitor navigation and accessibility for sight- and hearingimpaired visitors. Sign language, for example, is available through a dedicated app. 
Absent among enabling technologies was the evidence of the use of cloud computing platforms, although there are proposed smart cultural frameworks in the literature that include cloud platforms (Angelaccio et al. 2012, García-Crespo et al. 2016). Across the sample studies, it was difficult to determine use of cloud infrastructure due to the lack of available technical literature on the architecture of systems. In the cultural heritage domain, the Europeana Cloud is one of the larger cloud-based infrastructure projects in operation, hosting several million digital items, and supporting data services arising from the Europeana Open Data and associated programs.

IoT, as a nearly synonymous term with smart cities, remains an evolving technology, and has not reached an operational level of integration in smart cultural heritage, although there is potential for loT to underpin various smart cultural services (Chianese \& Piccialli 2014, Jara et al. 2015). The EU funded DATABENC (2014) initiative piloted an IoT service-oriented framework for an art exhibition of sculptures within the Maschio Angioino castle in Naples. Architectural components comprise an IoT enabled environment, with WSN and NFC subsystems (Amato et al. 2013, Chianese \& Piccialli 2014). More recently, an loT platform supporting sensors and mobile devices is being architected for Gallery One of the Cleveland Museum of Art in which The Collection Wall and ArtLens mobile app are key features (Alexander 2014).

\subsection{Visualisation technologies}

Forms of geovisualisation, from floor guides to location points and thematic maps, are pervasive and essential features of the applications and services across the selected case studies. This underpins a primary characteristic of smart environments, that of location-awareness relating to the user, place, and surrounding objects at any one time. Geovisualisation also reinforces other visualisation technologies such as AR which is bound to a location point and wayfinding activities.

3D visualisation, including computer generated objects, figured prominently in those examples with AR applications and immersive environments, such as PureLand 360 and Ai WeiWei 360, offering rich and layered forms of information. The digital 3D models in the preservation and reconstruction examples, Rekrei and Zamani, highlight that the protection of heritage and culture must remain a high priority for all cultures. These online collections of 3D reconstructions representing endangered or destroyed artefacts, cultural landmarks and monuments bring new resonance to the role that "virtual museums" can play in terms of knowledge and wider accessibility of cultural heritage (Styliani et al. 2009). The worldwide engagement of thousands of users supporting Rekrei's mission, in particular, also profiles the potential role of citizens in collectively protecting global cultural heritage, and that we do not need to be physically in the same place to participate in this goal.

The pervasiveness of $A R$ and/or $A R$ elements in the selected projects supports the adoption growth of this technology in the cultural heritage sector as a popular visualization paradigm, arising from tourism applications (Yovcheva 2012, Yovcheva et al. 2014) to educational and exhibition spaces (Cassella \& Coelho 2013, Garcia-Crespo 2016, Risseeuw et al. 2016). Museums, galleries and other cultural organisations have been trialling AR systems for several years, such as in the example of ARCHEOGUIDE, and the National Science Museum in Tokyo, in which AR technology was used to overlay "flesh" onto the dinosaur skeletons on display (Kondo et al. 2007). The Skin \& Bones AR app at the National Museum of Natural History has advanced this use, to bring dinosaur skeletons and fossils alive through a mix of $A R$, animation and gamification. The Museum has also provided the opportunity for children to use the AR app at home with a downloadable resource that simulates the museum experience.

The potential of AR in outdoor settings is exemplified by the Museum of London's highly successful StreetMuseum app that has been available as a downloadable app for over five years. The ROM Ultimate Dinosaur exhibition brought dinosaurs to life in the city of Toronto at bus shelters and public spaces with signposted instructions on how to activate the AR experience.

RecoVR Mosul and Ai WeiWei both use AR elements in that they use the real environment as a background with overlaid information on top. The applications are themselves accessible through web browsers as photorealistic $360^{\circ}$ panoramas, but alternatively can be experienced as 3D immersions in virtual reality (VR). The potential intersections of VR and smart environments are yet to be explored further.

\subsection{Other observations}

Over half of the case studies employed two or more enabling technologies and two or more visualization types. The high availability of mobile apps among most of the studies suggests that this an important criterion in making accessible more readily digital content and allowing users further flexibility in how, when and where they may want to access the content, and share it. Of the downloadable apps, all were available on iPhone or iPad, revealing, perhaps, that iPhone had an earlier start in the sector as a user engagement device. 
This also brings to the foreground a related and larger question of accessibility across the case studies. Rekrei and Zumani are two examples providing relatively low barriers to access content by a distributed audience. There is a means to directly contribute content as in the case of Rekrei. Of note both these projects are largely community sourced and fall outside of more established cultural heritage organisations. Community engagement in the co-design of smart and open services is a characteristic of participatory planning that is starting to gain traction in smart city agendas (Saunders \& Baeck 2015).

In most of the case examples audiences can interact with the content through native web browsers. In locations with a ready infrastructure, such as free Wi-Fi and/or WSN, accessibility is also increased. In the smart cities of London and Barcelona, the barrier is lowered for visitors to use digital trail apps around the city, such as StreetMuseum and Paisatge respectively, but interactivity options may be less, or less intense, compared to what might be achieved in an architected indoor environment, for example.

A final and related observation concerns the pervasiveness of personalization options for users in most of the case studies, for instance, to tag and share "favourites" as in the examples of the Collection Wall and The Pen. The ability to save digital trails and other content for a post visit experience (usually accessible through a web link) was a key feature in more than half of the case examples. This continuity of the user experience is an important one, and one that could potentially lead to insights into the development of smart cultural services.

\section{FUTURE OPPORTUNITIES}

There is clear evidence of the potential of cultural heritage organisations to play a significant role in the development of the fabric of smart cities. The sector already has a strong provenance with decades long experience in piloting emerging and embedded technologies relevant to smart city services, and in its understanding of user-centric interaction characterised by context-awareness, personalization and adaptation.

What appears to be lacking, thus far, are more visible pathways for cultural heritage organisations to participate in co-development and/or co-design of services that might be possible alongside smart city strategies. Perhaps the closest example of potential synergistic services are existing developments in digital tourism, but these still tend to be separate from a more integrated approach to city and wider services.

In the context of the present study, there remains a further gap in exploring a wider spread of organisations across geographies, specifically from Asia, South America and Africa that may point to new examples and perspectives on smart cultural heritage as well as the identification of new and smart service priorities.

The possibility of connecting across geographies of place and accessing cultural and citizen centric services made possible through cultural organisations has resonance with the idea of "a distributed museum" (Bautista \& Balsamo 2011).

No longer located in a particular physical space, the museum extends its presence through virtual spaces on the web as well as in the transient spaces created through the diverse practices and technologies of mobility.

This reinforces the importance of mobility in our thinking of future opportunities. To this we can add that open platforms and standards still need to be part of the discussion, and most critically we cannot lose sight that "smartness" is also about citizen centric approaches and how we can involve communities in achieving smarter cultural heritage.

\section{REFERENCES}

Alexander, J. (2014) Gallery One, the first year: sustainability, evaluation process, and a new smart phone app. MW2014: Museums and the Web. URL: http://mw2014.museumsandtheweb.com/paper/galleryone-the-first-year-sustainability-evaluation-process-anda-new-smart-phone-app/ (retrieved 30 March 2017).

Amato, F. et al. (2013) The talking museum project. In Procedia Computer Science, 4th International Conference on Emerging Ubiquitous Systems and Pervasive Networks (EUSPN-2013), 21(0), pp. 114-121.

Angelaccio, M. et al. (2012) Smart and mobile access to cultural heritage resources: a case study on ancient Italian Renaissance villas. In IEEE 21st International WETICE, pp. 310-314.

Angelidou, M. (2015) Smart cities: A conjuncture of four forces. Cities, 47, pp. 95-106.

Australian Government. (2016) Smart Cities Plan. Commonwealth of Australia. Canberra: Department of the Prime Minister and Cabinet. URL: https://cities.dpmc.gov.au/smart-cities-plan

(retrieved 5 April 2017).

Bautista, S. and Balsamo, A. (2011) Understanding the distributed museum: mapping the spaces of museology in contemporary culture. In J. Trant and D. Bearman (eds.), Museums and the Web 2011: Proceedings. Toronto: Archives \& Museum Informatics.

Bego, K. (2016) Pokémon Go and the marketing agencies of the augmented world. Nesta blog, 12 July. 
URL: $\quad$ http://www.nesta.org.uk/blog/pokemon-go-andmarketing-agencies-augmented-world (retrieved 30 March 2017).

Borda, A. and Bowen, J. P. (2011) Virtual collaboration and community. In Information Resources Management Association (ed.), Virtual Communities: Concepts, Methodologies, Tools and Applications. IGI Global, chapter 8.9, pp. 2600-2611.

Casella, G. and Coelho, M. (2013) Augmented heritage: situating augmented reality mobile apps in cultural heritage communication. In Proceedings of the 2013 International Conference on Information Systems and Design of Communication (ISDOC '13). ACM, New York, NY, USA, pp. 138-140. DOI: 10.1145/2503859.2503883

Chianese, A., Piccialli, F. and Valente, I. (2015) Smart environments and cultural heritage: a novel approach to create intelligent cultural spaces. Journal of Location Based Services, 9(3), pp. 209-234. DOI: 10.1080/17489725.2015.1099752

Chianese, A. and Piccialli, F. (2014) SmaCH: A framework for smart cultural heritage spaces. In Tenth International Conference on Signal-Image Technology \& Internet-Based Systems, pp. 477-484.

Cisco. (2014) Smart City Readiness: Understand the Issues to Accelerate the Journey. White Paper. Smart Cities Council. URL:

http://www.cisco.com/c/dam/m/en in/innovation/smartciti es/assets/white-paper-c11-732985.pdf (retrieved 5 April 2017).

DATABENC (2014) Distretto ad Alta Tecnologia per i Beni Culturali. Italy. URL: http://www.databenc.it/en/ (retrieved 30 March 2017).

EC (2015) Smart Cities and Communities: The European Innovation Partnership on Smart Cities and Communities. European Community, 4 June. http://ec.europa.eu/eip/smartcities/index en.htm (retrieved 24 April 2017).

Europeana (2015) Transforming the world with culture. White Paper, Europeana Foundation. September. URL: http://pro.europeana.eu/files/Europeana Professional/Pu blications/Europeana\%20Presidencies $\% 20$ White $\% 20 \mathrm{~Pa}$ per.pdf (retrieved 30 March 2017).

Filippini-Fantoni, S. and Bowen, J. P. (2007) Bookmarking in museums: Extending the museum experience beyond the visit? In Bearman, D. and Trant, J. (eds.), Museums and the Web 2007: Proceedings. Toronto: Archives \& Museum Informatics.

García-Crespo, A. et al. (2016) CESARSC: Framework for creating cultural entertainment systems with augmented reality in smart cities. Computer Science and Information Systems, 13(2), pp. 395-425. DOI: 10.2298/CSIS150620006G

Gartner (2017) Gartner says 8.4 Billion Connected "Things" will be in Use in 2017, up 31 percent from 2016. Newsroom, Press Release. Gartner Inc., 7 February. URL: $\quad$ http://www.gartner.com/newsroom/id/3598917 (retrieved 5 April 2017).

GrowSmarter (2015) GrowSmarter: transforming cities for a smart, sustainable Europe. GrowSmarter URL: http://www.grow-smarter.eu (retrieved 30 March 2017).

IEEE (2017) IEEE Smart Cities. IEEE. URL: http://smartcities.ieee.org (retrieved 30 March 2017).
ISO/IEC (2014) Information technology: Smart Cities, Preliminary Report. ISO/IEC JTC 1. URL: https://www.iso.org/iso/smart cities report-jtc1.pdf (retrieved 30 March 2017).

Jara, A. J. et al. (2015) Internet of Things for cultural heritage of smart cities and smart regions. In 29th International Conference on Advanced Information Networking and Applications Workshops, pp. 668-675. DOI: 10.1109/WAINA.2015.169

Kondo, T. et al. (2007) Mixed reality technology at a Natural History Museum. In Trant, J. and Bearman, D. (eds), Museums and the Web 2007: Proceedings. Toronto: Archives \& Museum Informatics.

Lagoudi, E. and Sexton, C. (2010) Old Masters at your fingertips: the journey of creating a museum app for the iPhone and iTouch. In Trant, J. and Bearman, D. (eds.), Museums and the Web 2010: Proceedings. Archives \& Museum Informatics.

$\mathrm{Li}$, K. et al. (2016) Categorisation of visualisation methods to support the design of Human-Computer Interaction Systems. Applied Ergonomics, 55, pp 85107. DOI: $10.1016 / \mathrm{j}$. apergo.2016.01.009

Nokia (2017) Enabling smart, safe and sustainable cities: Strategic white paper. Nokia Corporation. URL: http://resources.alcatel-lucent.com/asset/191721 (retrieved 30 March 2017).

Palomar, E., Chen, X., Liu, Z., Maharjan, S., and Bowen, J. P. (2016) Component-based modelling for scalable smart city systems interoperability: A case study on integrating energy demand response systems. Sensors, 16(11):1810, 2016. DOI: $10.3390 / \mathrm{s} 16111810$

Risseeuw, M. et al. (2016) Authoring augmented digital experiences in museums. In Buono, P. et al. (eds), AVI '16: Proceedings of the International Working Conference on Advanced Visual Interfaces. New York, ACM, pp. 340-341.

Roffia, L. et al. (2005) Context awareness in mobile cultural heritage applications. In Smart Environments and their Applications to Cultural Heritage Workshop. 7th International Conference on Ubiquitous Computing (UBICOMP05), Tokyo, Japan, pp. 33-36.

Saunders, T. and Baeck, P. (2015) Rethinking the Smart Cities from the Ground Up. June 2015. London: Nesta.

Soja, E. and Kanai, E. (2006) The Urbanization of the World. In Burdett, R. and Sudjic, D. (eds), The Endless City. London: Phaidon Books.

Styliani, S. et al. (2009) Virtual museums, a survey and some issues for consideration. Journal of Cultural Heritage, 10, pp. 520-528.

Vlahakis, V. et al. (2001) ARCHEOGUIDE: First results of an Augmented Reality, Mobile Computing System in Cultural Heritage Sites. In Proceedings of the Conference on Virtual Reality, Archaeology, and Cultural Heritage (VAST'01), Glyfada, Greece, 28-30 November, pp.131-140.

Yovcheva, Z., Buhalis, D., and Gatzidis, C. (2012) Overview of smartphone augmented reality applications for tourism. e-Review of Tourism Research (eRTR), 10(2), pp. 63-66.

Yovcheva, Z. et al. (2014) Empirical evaluation of smartphone augmented reality browsers in an urban tourism destination context. International Journal of Mobile Human Computer Interaction, 6(2), April-June, pp. 10-31. 